\title{
Asociación entre tamización de cáncer de próstata, vinculación al sistema de salud y factores asociados en adultos mayores: análisis secundario de la encuesta SABE Bogotá, Colombia
}

\section{Relationship between prostate cancer screening, linkage to the health system and associated factors in older adults from Bogotá, Colombia: Secondary analysis of the SABE survey}

\author{
Miguel Germán Borda1,2,3 David Gabriel David-Pardo ${ }^{1,2}$ Julián David Ríos-Zuluaga1 \\ Ana Isabel López-Zea ${ }^{4}$ Laura Margarita Forero-Borda ${ }^{3}$ Santiago Gutiérrez ${ }^{1}$ \\ Carlos Alberto Cano-Gutiérrez ${ }^{1,2,3}$
}

\footnotetext{
1 Semillero de Neurociencias y Envejecimiento, Facultad de Medicina, Pontificia Universidad Javeriana, Bogotá, Colombia

2 Instituto de Envejecimiento, Pontificia Universidad Javeriana,

Bogotá, Colombia

3 Unidad de Geriatría, Hospital Universitario San Ignacio,

Bogotá, Colombia

${ }^{4}$ Facultad de Medicina, Universidad del Rosario, Bogotá, Colombia
}
Address for correspondence Miguel Germán Borda, Semillero de Neurociencias y Envejecimiento, Facultad de Medicina, Pontificia Universidad Javeriana, Bogotá, Colombia (e-mail: mmborda@gmail.com).

Urol Colomb 2018;27:35-41.

\author{
Resumen \\ Palabras Clave \\ - cáncer de próstata \\ - tamización \\ - anciano \\ - colombia \\ - epidemiología \\ - prevalencia
}

Introducción y objetivos El cáncer de próstata es una enfermedad con alta prevalencia en nuestra población. Su diagnóstico temprano es importante para mejorar el pronóstico de esta enfermedad. El objetivo de este artículo es evidenciar los factores asociados a la oportunidad de tamización en adultos mayores de la ciudad de Bogotá, Colombia.

Métodos Se analizaron los datos del estudio Salud, Bienestar y Envejecimiento (SABE) Bogotá 2012, que incluyó a 736 hombres de 60 años o más. Se utilizó como variable dependiente el autorreporte de examen de próstata en los 2 últimos años y se evaluó con respecto a factores sociodemográficos por medio de un análisis multivariado.

Resultados Se encontró una prevalencia de cáncer de próstata del 3,15\%. El 57,8\% de los hombres se habían realizado al menos un examen de próstata. Aquellos afiliados al régimen contributivo tenían mayor oportunidad para ser tamizados con respecto a los vinculados/no asegurados (OR: 8,81) (IC95\%: 2,92-26,63) ( $\mathrm{p}<0,001)$, al igual que los del régimen subsidiado (OR: 3,70) (IC95\%; 1,20-11,41) $(\mathrm{p}=0,023)$. received

November 18, 2016

accepted

February 28, 2017

published online

January 10, 2018
DOI https://doi.org/

10.1016/j.uroco.2017.02.005.

ISSN 0120-789X.

eISSN 2027-0119.
Copyright ( 2018, Sociedad Colombiana License terms de Urología. Publicado por Thieme Revinter Publicações Ltda., Rio de Janeiro, Brazil. Todos los derechos reservados. 


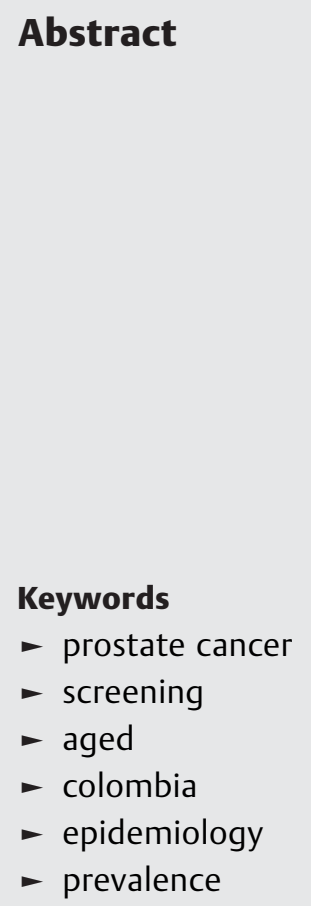

Conclusión Existe una inequidad en cuanto a la oportunidad de tamización de cáncer de próstata según el tipo de seguridad social, por lo que se deben optimizar las estrategias de detección temprana que fortalezcan la inclusión de toda la población. Se necesitan más estudios que brinden mayor información sobre esta problemática.

Introduction Prostate cancer is a high prevalence disease in our male population. Early diagnosis is important in order to improve its prognosis. The aim of this article is to describe the factors associated with prostate cancer screening of older adults in Bogotá, Colombia.

Materials and methods The study used data from the Bogotá 2012 Health, WellBeing, and Ageing (Salud, Bienestar y Envejecimiento [SABE]) survey, which included 736 men aged 60 years or older. The dependent variable used was self-reported prostate screening in the last 2 years. An analysis was performed to determine the between this variable and socio-demographic variables and comorbidities using multivariate analysis.

Results There was a prevalence of prostate cancer of $3.15 \%$, with $57.8 \%$ of the population having had at least one prostate examination. Those affiliated to a health insurance scheme were more likely to be screened than the uninsured with an OR: 8.81 , 95\% Cl: $2.92-26.63, P<.001$, as those affiliated to subsidized social security health scheme OR: $3.70,95 \% \mathrm{Cl}: 1.20-11.41, P=.023$, respectively).

Conclusion There is inequity in the opportunity of screening for prostate cancer according to the type of health insurance scheme. Early detection strategies must be strengthened in order to include the entire population. Further studies are needed to provide more information on this issue.

\section{Introducción}

Debido al fenómeno de transición demográfica, ${ }^{1}$ la cantidad de enfermedades dependientes de la edad han aumentado. En los hombres las enfermedades asociadas a la próstata tienen un papel fundamental, dada su alta prevalencia y carga de enfermedad. ${ }^{2}$

El cáncer de próstata constituye un problema de salud pública por su alto impacto en cuanto a frecuencia, mortalidad, y costos, ${ }^{3,4}$ siendo a nivel mundial el segundo tipo de cáncer más diagnosticado en hombres y la quinta causa de muerte masculina por cáncer. ${ }^{5}$ La proyección mundial es de 1,7 millones de casos nuevos para el año 2030 , con una mortalidad esperada de 499.000 casos; ${ }^{6}$ en los EE. UU. es el cáncer visceral más diagnosticado, estimándose para el 2016 un aproximado de 180.890 nuevos casos y 20.120 muertes por esta causa. ${ }^{7}$ En Colombia, el cáncer de próstata representa la primera causa de cáncer según prevalencia e incidencia y la segunda causa de mortalidad por cáncer en la población masculina. ${ }^{2,8}$ Se estiman $6.500-$ 8.000 casos nuevos de cáncer de próstata por cada año en el país (tasa de incidencia ajustada por edad de 40,5-45,9/ 100.000 hombres), con una tendencia al alza con respecto a los últimos años de 22,3 hasta 64,8/100.000 habitantes. ${ }^{2}$

La introducción de métodos de detección precoz y el uso de varias técnicas en conjunto puede explicar el aumento de la incidencia del cáncer de próstata. La técnica más usada es la denominada tamización de oportunidad, que conjuga el antígeno prostático específico y el tacto rectal en hombres mayores de 50 años, asintomáticos, que acuden a consulta médica por diferentes causas. ${ }^{2,9,10}$ Se estima que la sensibilidad y la especificidad del antígeno prostático específico son del $72,1 \%$ y del $93,2 \%$, respectivamente; y del tacto rectal del 53,2\% y del 86,6\%. Por lo anterior, se propone su uso en escenarios clínicos, en lugar de programas poblacionales. $^{2,10}$ La tamización, que ha demostrado tener un impacto importante en el diagnóstico, ${ }^{11,12}$ es controvertida en cuanto a su efectividad en la reducción de mortalidad por cáncer de próstata. 2,13-15

Ante el aumento del cáncer en el perfil epidemiológico de los colombianos, es preciso realizar de forma oportuna $\mathrm{y}$ sistematizada intervenciones que generen un alto impacto poblacional e individual. Por esta razón, el Ministerio de Salud y Protección Social a partir de la resolución 1442 del 2013, adopta la Guía de Práctica Clínica para la detección oportuna, diagnóstico, tratamiento, seguimiento $\mathrm{y}$ rehabilitación de pacientes con cáncer de próstata. ${ }^{2}$ Sin embargo, en términos prácticos, la aplicación de estas intervenciones es difícil por la variedad de población en cuanto al nivel socioeconómico, escolaridad y acceso al sistema de salud. ${ }^{16-19}$ Ante esto, se busca fortalecer la implementación de programas de tamización que permitan reunir más información al respecto y evaluar sus resultados posteriores. 
El objetivo del presente trabajo es dilucidar la situación actual de la tamización de cáncer de próstata en Bogotá, Colombia, incluyendo su asociación con factores como edad, escolaridad, estrato socioeconómico, depresión, autopercepción del estado de salud y tipo de seguridad social.

\section{Materiales y métodos}

Se hizo un análisis secundario de la encuesta Salud, Bienestar y envejecimiento en América Latina y el Caribe (SABEBogotá), que se llevó a cabo en el año 2012, en la zona rural y urbana de la ciudad de Bogotá. La muestra está conformada por 2.000 personas $\geq 60$ años de edad, la cual corresponde a una muestra estadísticamente representativa con un diseño muestral que tuvo en cuenta: a) un análisis de la población $\geq 60$ años de edad; b) el universo de análisis definido como la población mayor de la ciudad; b) el censo nacional realizado por el DANE para el año 2005; c) muestreo probabilístico de conglomerados considerado en 3 etapas: la primera radios censales o segmentos, la segunda viviendas y la tercera personas; y d) la muestra estratificada con criterios geográficos, demográficos, o socioeconómicos. Los cálculos se realizan con las fórmulas correspondientes al tipo de diseño muestral, teniendo en cuenta un $5 \%$ de error relativo, que permite estimaciones confiables para las desagregaciones geográficas (urbano y rural) y temáticas incluidas en la encuesta SABE.

La cobertura total fue del $81,9 \%$, con un rechazo inferior al $20 \%$. El cuestionario ha sido aplicado en múltiples ciudades de Latinoamérica y en el caso de Bogotá se adaptó y ajustó a las características de su población, sin perder la capacidad de ser comparado.

Para la recolección de datos, se asignaron equipos de trabajo de campo conformados por un supervisor, 3 o 4 encuestadores y un antropometrista. Los equipos fueron entrenados por los investigadores principales, investigadores temáticos, estadístico y coordinador de campo. El 11,7\% de las personas mayores seleccionadas en la muestra SABE-Bogotá tuvieron un informante proxy para responder la encuesta debido a limitaciones físicas o mentales.

Los datos recolectados fueron digitados y grabados en Excel para Windows. El 11,7\% de las personas mayores seleccionadas en la muestra SABE-Bogotá tuvieron un informante proxy para responder la encuesta.

El protocolo de investigación cumple con los principios éticos estipulados en la Declaración de Helsinki de 1975; fue aprobado por el Comité de Investigación y Ética de la Pontificia Universidad Javeriana y la Oficina de Investigación del Hospital Universitario San Ignacio. El consentimiento informado fue obtenido de cada persona que participó.

\section{Variables}

\section{Variables dependientes}

Como variable dependiente se incluyó el autorreporte de realización de examen de próstata, el cual se evaluó con la pregunta: En los últimos 2 años: ¿Alguna vez se ha hecho un examen de próstata?

\section{Variables independientes}

Como variables independientes se incluyeron variables sociodemográficas, depresión, percepción del estado de salud actual, tipo de seguridad social y la presencia o no de cáncer de próstata, muchas de las cuales categorizamos en aras de facilitar el análisis y la comprensión de los resultados.

Las variables sociodemográficas incluidas fueron: sexo, edad, escolaridad y estrato socioeconómico. La edad la agrupamos en los siguientes grupos de años: 60-65, 66-70, 71-75 y 75 o más años. Para la escolaridad, realizamos 4 grupos teniendo en cuenta los años de estudio que se realizan en primaria, bachillerato y luego de finalizar el colegio: 0, 1-5, 6-10 y 11 o más años de estudio. El estrato socioeconómico lo categorizamos en 3 grupos: 1 y 2 (bajo), 3 y 4 (medio) y 5 y 6 (alto).

La depresión del adulto mayor fue evaluada mediante la Escala de Depresión Geriátrica de Yesavage (GDS, por sus siglas en inglés), que permitía obtener de 0 a 15 puntos. Se tomó como ausencia de depresión si el individuo lograba de 0-5 puntos, mientras que si lograba 6 puntos o más había presencia de depresión. ${ }^{20}$ Así mismo, incluimos la percepción del estado de salud a través de la pregunta En cuanto a su salud, ¿diría usted que es: excelente, muy buena, buena, regular, mala; por lo que decidimos conformar 2 grupos: uno que incluía las 3 primeras respuestas y otro que incluía las 2 últimas. Además, se interrogó acerca del tipo de seguridad social a la que pertenece (contributivo, subsidiado, vinculado o no asegurado). Por último, se dilucidó sobre la presencia o no de cáncer de próstata mediante la pregunta de autorreporte ¿Alguna vez un médico le ha dicho si tiene cáncer o un tumor maligno? y, si la persona respondía afirmativo, se le preguntaba: ¿Para qué tipo de cáncer recibió tratamiento?, entre cuyas opciones de respuesta se encontraba «Próstata» (-Fig. 1).

Si bien una gran cantidad de datos fueron extraídos con la modalidad de autorreporte, para el control de sesgo de memoria se intentaron usar estrategias como preguntar o corroborar información con el familiar o acompañante (proxy), pedir cajas de medicamentos o revisar registros de exámenes o citas recientes.

Este proyecto fue financiado por el Departamento Administrativo de Ciencia, Tecnología e Innovación-Colciencias, código 120354531692 y por la Pontificia Universidad Javeriana.

\section{Análisis estadístico}

Se hallaron las frecuencias simples y porcentajes de todas las variables estudiadas, ya que todas eran categóricas. Luego, realizamos análisis bivariados mediante pruebas de chi-cuadrado para determinar la asociación entre la variable dependiente (tamización de cáncer de próstata) y las independientes. Por último, efectuamos un análisis multivariado (procedimiento stepwise) incluyendo las variables que fueron estadísticamente significativas en el análisis bivariado, prediciendo los odds ratio (OR) de las variables de interés que favorecieran o no la realización del examen de próstata. El nivel de significación estadística fue fijado en $\mathrm{p}<0,05$. Los datos fueron analizados usando el paquete estadístico STATA (versión 12 para Macintosh). 


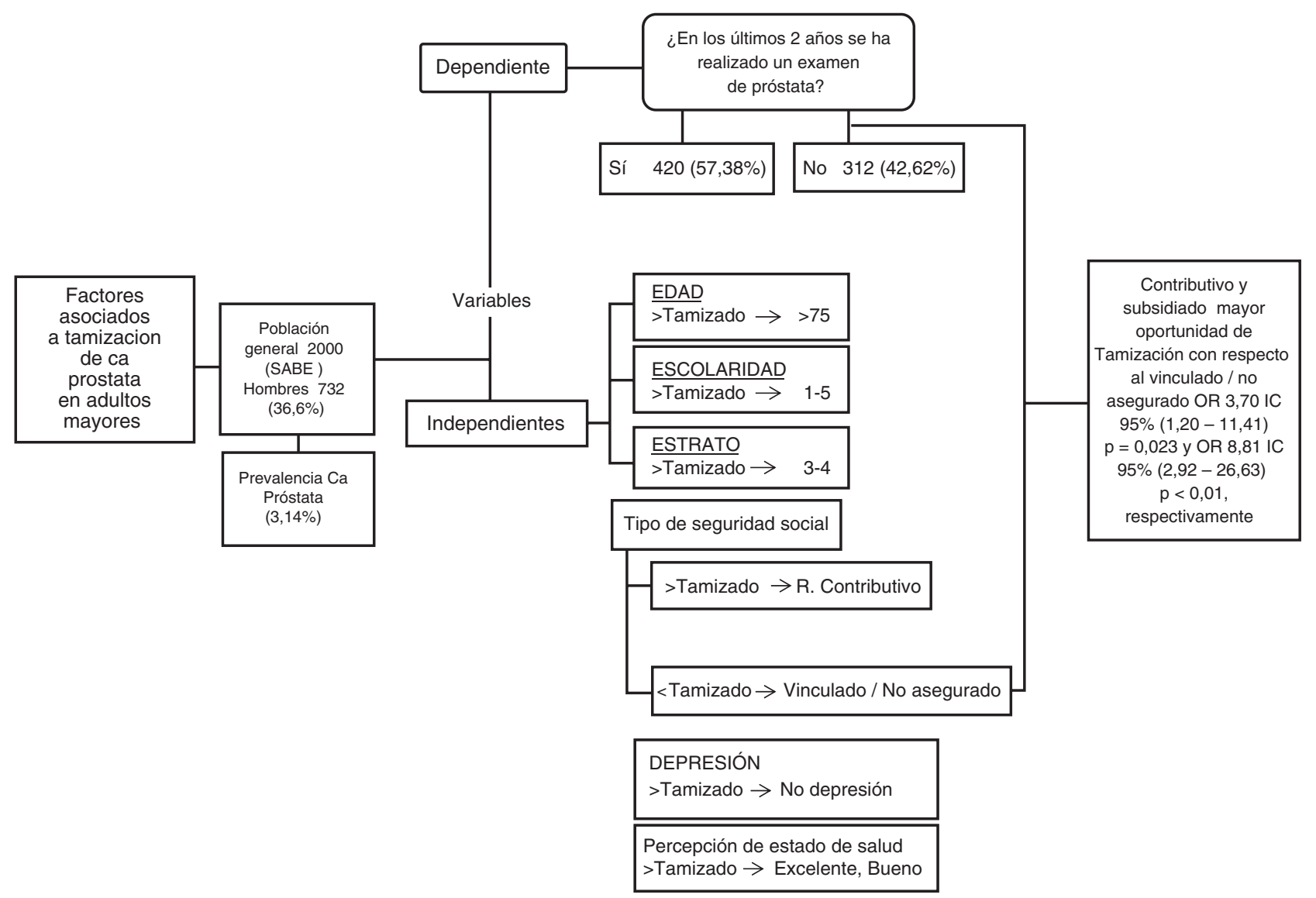

Fig. 1 Variables tenidas en cuenta para el análisis de datos y resultados principales.

\section{Resultados}

\section{Datos generales de la población}

Del total de 2.000 personas encuestadas con el instrumento $\mathrm{SABE}$, un 36,6\% (732) eran hombres, de los cuales el $28,28 \%$ tenían entre 60 y 65 años, el 21,72\% entre 66 y 70, el 19,40\% entre 71 y 75 años y el 30,60\% eran mayores de 75 años, siendo este el grupo de edad más prevalente. El grado de escolaridad medido en años completados de estudio se organizó en 4 grupos de la siguiente manera: 0 años de estudios con un 7,24\%, 1-5 años con un 55,87\%, 6-10 años representaban el $14,34 \%$ y mayor o igual a 11 años el 22,54\%; siendo el intervalo entre 1-5 años el más prevalente. En cuanto al estrato socioeconómico, los estratos 1 y 2 representaban el 54,51\%, los estratos 3 y 4 el 42,35\% y los estratos 5 y 6 el 3,14\%, siendo más prevalente el conformado por los estratos más bajo.

Dentro de la variable percepción del estado de salud, obtuvimos que un 51,50\% consideraba que su salud en ese momento era excelente, buena o muy buena, mientras que el $48,50 \%$ la percibía como regular o mala. Con respecto al tipo de seguridad social, el 3,28\% eran vinculados o no asegurados, el $27,46 \%$ pertenecía al régimen subsidiado y el $69,26 \%$ al régimen contributivo, siendo este último grupo el más prevalente.

A la pregunta En los últimos 2 años, ¿alguna vez se ha hecho un examen de próstata? se obtuvo que 420 hombres (57,2\%) se habían realizado al menos un examen de próstata y se encontró una prevalencia de cáncer de próstata del 3,14\% (- Tabla 1 ).

\section{Factores asociados a la tamización de próstata:} Análisis bivariado

La tamización es más prevalente en las personas que tienen de 1 a 5 años de escolaridad ( $p<0,001$ ). De acuerdo a estratos socioeconómicos, en el 1 y 2 el 47,38\% se tamizaron; en el 3 y 4 el 48,33\%; y el grupo de estratos 5 y 6 el 4,29\% (p < 0,001).

Evidenciamos prevalencia de tamización más baja en personas con depresión (18,57\%) que sin depresión $(81,43 \%)(p=0,016)$. También se encontró una prevalencia más alta en personas con régimen contributivo de seguridad social ( 80 vs. $19,05 \%$ en régimen subsidiado vs. $0,95 \%$ en vinculados o no asegurados con $\mathrm{p}<0,001$ ) (-Tabla 2).

\section{Análisis multivariado}

En el análisis multivariado encontramos que las personas pertenecientes al régimen subsidiado tenían un riesgo mayor de ser tamizados con un OR: 3,70 (IC95\%: 1,20-11,41) $(\mathrm{p}=0,023)$ al igual que las del régimen contributivo con un OR: 8,81 (IC95\%: 2,92-26,63) ( $\mathrm{p}<0,001$ ) con respecto a aquellos hombres en el régimen vinculado o no asegurados ( - Tabla 3 ).

\section{Discusión}

El presente estudio constituye un acercamiento a la situación actual de la tamización del cáncer de próstata en los adultos mayores residentes de Bogotá, Colombia. Encontramos que el 
Tabla 1 Aspectos generales de la población $(n=732)$

\begin{tabular}{|c|c|c|}
\hline Variables & Frecuencia & $\%$ \\
\hline \multicolumn{3}{|l|}{ Edad } \\
\hline $60-65$ & 207 & 28,28 \\
\hline $66-70$ & 159 & 21,72 \\
\hline $71-75$ & 142 & 19,40 \\
\hline$\geq 75$ & 224 & 30,60 \\
\hline \multicolumn{3}{|l|}{ Escolaridad (años) } \\
\hline 0 & 53 & 7,24 \\
\hline $1-5$ & 409 & 55,87 \\
\hline $6-10$ & 105 & 14,34 \\
\hline$\geq 11$ & 165 & 22,54 \\
\hline \multicolumn{3}{|l|}{ Estrato socioeconómico } \\
\hline $1-2$ & 399 & 54,51 \\
\hline $3-4$ & 310 & 42,35 \\
\hline $5-6$ & 23 & 3,14 \\
\hline \multicolumn{3}{|l|}{ Depresión (Yesavage) } \\
\hline $0-5$ (ausencia) & 573 & 78,28 \\
\hline$\geq 6$ (presencia) & 159 & 21,72 \\
\hline \multicolumn{3}{|l|}{ Percepción estado de salud } \\
\hline Bueno, muy bueno, excelente & 377 & 51,50 \\
\hline Regular, malo & 355 & 48,50 \\
\hline \multicolumn{3}{|l|}{ Tipo de seguridad social } \\
\hline Contributivo & 507 & 69,26 \\
\hline Subsidiado & 201 & 27,46 \\
\hline Vinculado o no asegurado & 24 & 3,28 \\
\hline \multicolumn{3}{|c|}{ ¿Alguna vez se ha hecho un examen de próstata? } \\
\hline Sí & 420 & 57,38 \\
\hline \multicolumn{3}{|l|}{ Cáncer de próstata } \\
\hline Sí & 23 & 3,14 \\
\hline
\end{tabular}

$57,38 \%$ de los adultos mayores hombres alguna vez en su vida se ha realizado un examen de próstata. Este porcentaje es bajo comparado con la recomendación de tamizar a todo adulto mayor de 50 años, asintomático, que acuda a consulta médica por diferentes causas y comparado a la meta nacional de lograr coberturas hasta en un $70 \%{ }^{2}$ Como posibles explicaciones de este resultado, planteamos que puede ser debido a falta de conocimiento del personal médico ante esta recomendación, difícil acceso de la población adulta mayor a los servicios de salud o cuestiones culturales como ciertas creencias que llevan a que el paciente se niegue a la realización de un tacto rectal.

La prevalencia del cáncer de próstata encontrada fue del $3,14 \%$, alta si la comparamos con la estimada en la $V$ edición del informe técnico del Observatorio Nacional de Salud en donde reportan una prevalencia en Colombia del 0,146; 0,153; 0,160; 0,168 y 0,176\% para los años 2010, 2011, 2012, 2013 y 2014, respectivamente. ${ }^{8}$ Sin embargo, esta
Tabla 2 Factores asociados a la tamización de cáncer de próstata: Análisis bivariado

\begin{tabular}{|c|c|c|}
\hline Variable & $\mathrm{N}(\%)$ & $\mathrm{p}$ \\
\hline \multicolumn{3}{|l|}{ Edad } \\
\hline $60-65$ & $114(27,14)$ & \\
\hline $66-70$ & $102(24,29)$ & \\
\hline $71-75$ & $84(20)$ & \\
\hline$\geq 75$ & $120(28,57)$ & 0,176 \\
\hline \multicolumn{3}{|l|}{ Escolaridad } \\
\hline 0 años & $23(5,48)$ & \\
\hline $1-5$ años & $207(49,29)$ & \\
\hline $6-10$ años & $68(16,19)$ & \\
\hline$\geq 11$ & $122(29,05)$ & $<0,001^{*}$ \\
\hline \multicolumn{3}{|l|}{ Estrato socioeconómico } \\
\hline $1-2$ & $199(47,38)$ & \\
\hline $3-4$ & $203(48,33)$ & \\
\hline $5-6$ & $18(4,29)$ & $<0,001^{*}$ \\
\hline \multicolumn{3}{|l|}{ Depresión (Yesavage) } \\
\hline 0-5 (ausencia) & $342(81,43)$ & \\
\hline$\geq 6$ (presencia) & $78(18,57)$ & 0,016 \\
\hline \multicolumn{3}{|l|}{ Percepción de estado de salud } \\
\hline Bueno, muy bueno, excelente & $223(53,10)$ & \\
\hline Regular, malo & $197(46,90)$ & 0,317 \\
\hline \multicolumn{3}{|l|}{ Tipo de seguridad social } \\
\hline Contributivo & $336(80)$ & \\
\hline Subsidiado & $80(19,05)$ & \\
\hline Vinculado o no asegurado & $4(0,95)$ & $<0,001^{*}$ \\
\hline
\end{tabular}

*Diferencias estadísticamente significativas.

alta prevalencia en nuestro estudio se debe principalmente a que la población es de alto riesgo (mayores de 60 años), mientras que la del Observatorio Nacional de Salud está en un rango de 15 años en adelante. Además, este resultado puede corresponder a la falta de programas de tamización poblacional efectivos y asequibles para el diagnóstico precoz de cáncer de próstata en todos los grupos etarios en Colombia. Estudios en otros países del mundo con sistemas de salud diferentes evidencian una relación directamente proporcional de mayor tamización cuanto mayor acceso a la atención médica. ${ }^{17}$

Un elemento llamativo que encontramos es la diferencia de oportunidad de tamización con respecto al tipo de seguridad social al que pertenece la persona, independientemente de la escolaridad, el estrato social y la presencia de depresión. Aquellos que pertenecen al régimen subsidiado tienen casi 4 veces más oportunidad de tamización que el vinculado, mientras que aquellos pertenecientes al régimen contributivo tienen casi 9 veces más oportunidad de tamización. Este escenario de inequidad preocupa en la medida en que la mayor parte de la población tiene una menor oportunidad de 
Tabla 3 Factores asociados a la tamización de cáncer de próstata: Análisis multivariado

\begin{tabular}{|l|l|l|l|}
\hline Variable & OR & IC 95\% & P \\
\hline Escolaridad & 1 & & \\
\hline 0 años & 0,96 & $0,52-1,77$ & 0,907 \\
\hline $1-5$ años & 1,63 & $0,80-3,34$ & 0,174 \\
\hline $6-10$ años & 1,95 & $0,95-4$ & 0,067 \\
\hline$\geq 11$ años & 1 & & \\
\hline Estrato social & 1,30 & $0,92-1,83$ & 0,141 \\
\hline $1-2$ & 1,62 & $0,56-4,68$ & 0,369 \\
\hline $3-4$ & \multicolumn{5}{|l|}{} \\
\hline 5-6 & 0,96 & $0,65-1,41$ & 0,828 \\
\hline Depresión (Yesavage) & \multicolumn{5}{|l|}{} \\
\hline 6-15 (presencia) & 1 & $1,20-11,41$ & $0,023^{*}$ \\
\hline Seguridad social & 3,70 & $2,92-26,63$ & $<0,01^{*}$ \\
\hline Vinculado o ninguno & 8,81 & 2 \\
\hline Subsidiado &
\end{tabular}

IC: intervalo de confianza; OR: odds ratio.

*Diferencias estadísticamente significativas.

acceso a los servicios de tamización, y esto se ve reflejado en la alta incidencia de cáncer de próstata encontrada. Todo esto se traduce a que por más que se plantee un excelente programa de tamización, si la mayor parte de la población no puede acceder a este, las consecuencias de la detección tardía de cáncer de próstata seguirán en aumento.

Por último, si bien la tamización de cáncer de próstata sigue siendo controversial en cuanto a efectividad, ${ }^{14,15}$ los resultados de este estudio pueden ser un reflejo de la situación actual del país en cuanto al acceso a diferentes programas de tamización. Preocupa el menor acceso de los regímenes subsidiado y vinculado, con respecto al contributivo. También cabe resaltar el papel del médico general en la importancia de realizar, a quien se indique, la tamización de cáncer de próstata y la oportuna remisión al urólogo para la instauración de un adecuado tratamiento. Además, sobresale el papel de los creadores de políticas públicas ya que es importante la generación de medidas que aumenten la oportunidad de tamización en la población.

Este estudio tiene ciertas limitaciones. Primero, al ser un estudio de corte transversal no fue posible determinar la causalidad de las asociaciones. Segundo, la variable dependiente es de autorreporte, lo que puede llevar a sesgo de memoria, así como la no discriminación del tipo específico de tamización (antígeno prostático específico, tacto rectal). De igual manera, este estudio tiene importantes fortalezas. En nuestro conocimiento, es uno de los pocos en Colombia que evalúa la asociación entre tamización de cáncer de próstata y determinantes sociales y comorbilidades en adultos mayores, además de determinar la prevalencia del cáncer de próstata en Bogotá, Colombia.

\section{Conclusiones}

El cáncer de próstata es una enfermedad prevalente en la población adulta mayor y su importancia radica en que la detección precoz por medio de programas poblacionales puede generar una disminución en la mortalidad, la morbilidad y costos en servicios de salud. Existe una inequidad en cuanto a la oportunidad de tamización de cáncer de próstata según el tipo de seguridad social, por lo que se hace necesario la creación de políticas públicas para optimizar las estrategias de detección temprana que fortalezcan la inclusión de toda la población en riesgo a los programas de tamización.

Se requieren más estudios que brinden mayor información sobre esta problemática.

\section{Responsabilidades éticas}

\section{Protección de personas y animales}

Los autores declaran que para esta investigación no se han realizado experimentos en seres humanos ni en animales.

\section{Confidencialidad de los datos}

Los autores declaran que han seguido los protocolos de su centro de trabajo sobre la publicación de datos de pacientes.

\section{Derecho a la privacidad y consentimiento informado}

Los autores han obtenido el consentimiento informado de los pacientes y/o sujetos referidos en el artículo. Este documento obra en poder del autor de correspondencia.

\section{Financiación}

Este proyecto ha sido financiado por el Departamento Administrativo de Ciencia, Tecnología e innovaciónColciencias, Código 120354531692 y por la Pontificia Universidad Javeriana.

Conflicto de intereses

Declaramos no poseer conflictos de intereses.

\section{Bibliografía}

1 Lee R. The demographic transition: Three centuries of fundamental change. J Econ Perspect [Internet] 2003;17(04):167-190

2 Cataño JG. Guía de práctica clínica para la detección temprana, diagnóstico, tratamiento, seguimiento y rehabilitación del cáncer de próstata, Colombia. Inst Nac Cancerol INC-ESE, COLCIENCIAS, Minist Salud y Protección Soc. 2013

3 Laviana AA, Ilg AM, Veruttipong D, Tan H-J, Burke MA, Niedzwiecki $\mathrm{DR}$, et al. Utilizing time-driven activity-based costing to understand the short- and long-term costs of treating localized, low-risk prostate cancer. Cancer 2016;122(03):447-455

4 Luis J, Matiz P, Julián N, Reyes A, Paula M, Becerra S, et al. Evolución de la mortalidad por cáncer de próstata en Colombia: estudio ecológico. Urol Colomb 2014;23(01):3-10

5 Torre LA, Bray F, Siegel RL, Ferlay J, Lortet-Tieulent J, Jemal A. Global cancer statistics, 2012. CA Cancer J Clin [Internet] 2015;65 (02):87-108

6 Center MM, Jemal A, Lortet-Tieulent J, Ward E, Ferlay J, Brawley O, et al. International variation in prostate cancer incidence and mortality rates. Eur Urol [Internet] 2012;61(06):1079-1092 
7 Freedland S. Measurement of prostate-specific antigen-UpToDate [Internet]. 2016. [consultado 2 Nov 2016]. Disponible en: https:// www.uptodate.com/contents/measurement-of-prostate-specificantigen

8 Castañeda Orjuela CA, Chaparro Narváez PE, Cotes Cantillo KP, Vargas Sandoval GA, Díaz Jiménez DP, Cárdenas Cárdenas LM, et al. Carga de enfermedad por enfermedades crónicas no transmisibles y discapacidad en Colombia. Obs Nac Salud. 2015; V edición

9 Alteri R, Bertaut TBD. Prostate cancer. Cancer Facts \& Figures Annual Bulletin. Am Cancer Soc Natl Heal Counc 2016:20-21

10 Mistry K, Cable G. Meta-analysis of prostate-specific antigen and digital rectal examination as screening tests for prostate carcinoma. J Am Board Fam Pract 2003;16(02):95-101

11 Ilic D, Neuberger MM, Djulbegovic M, Dahm P. Screening for prostate cancer. En: Ilic D, editor. Cochrane Database of Systematic Reviews. Chichester, UK: John Wiley \& Sons, Ltd; 2013. [consultado 26 Oct 2016]. Disponible en: http://doi.wiley. com/10.1002/14651858.CD004720.pub3

12 Schröder FH, Hugosson J, Roobol MJ, Tammela TLJ, Ciatto S, Nelen $\mathrm{V}$, et al. Prostate-cancer mortality at 11 years of follow-up. N Engl J Med 2012;366(11):981-990

13 LAG, Eisner MP, Kosary CL. SEER Cancer Statistics Review, 19731999. Natl Cancer Institute, Bethesda. 2002
14 Brett AS, Ablin RJ. Prostate-cancer screening-What the U.S. Preventive Services Task Force left out. N Engl J Med 2011;365 (21):1949-1951

15 Andriole GL, Crawford ED, Grubb RL, Buys SS, Chia D, Church TR, et al. Mortality results from a randomized prostate-cancer screening trial. N Engl J Med 2009;360(13):1310-1319

16 Pereira de Paiva E, Catarina Salvador da Motta M, Harter Griep R. Barreras en relación a los exámenes de rastreo de cáncer de próstata. Rev Latino-Am Enferm. 2011. [consultado 11 Nov 2016]. Disponible en: www.eerp.usp.br/rlae

17 Mitchell J. Examining the influence of social ecological factors on prostate cancer screening in urban African-American men. Soc Work Health Care 2011;50(08):639-655

18 Jeihooni AK, Kashfi SM, Kashfi SH, Heydarabadi AB, Imanzad M, Hafez AA. Factors associated with prostate cancer screening behavior among men over 50 in Fasa, Iran, based on the PRECEDE model. Electronic Physician 2015;7(02):1054-1062

19 Woods VD, Montgomery SB, Herring RP, Gardner RW, Stokols D. Social ecological predictors of prostate-specific antigen blood test and digital rectal examination in black American men. J Natl Med Assoc 2006;98(04):492-504

20 Yesavage JA, Brink TL, Rose TL, Lum O, Huang V, Adey M, et al. Development and validation of a geriatric depression screening scale: a preliminary report. J Psychiatr Res 1982;17(01):37-49 